\title{
Comparison of chemical restraint techniques in ostrich (Struthio camelus)
}

— Author(s)
Ciboto R ${ }^{1}$
Cortopassi SRG
Lopes MAE
Carvalho RC
Baitelo CG
Faculdade de Medicina Veterinária e
Zootecnia
Universidade de São Paulo

\section{Mail Address}

Rodrigo Ciboto

Rua Zina, 121

Jardim das Flores

06112-090. Osasco, SP, Brazil

E-mai: rodrigo@avtruz.com.br

\section{Keywords}

Anesthesia; chemical restraint; muscular relaxation; ostrich
Apoio:

Fundação de Amparo à Pesquisa do Estado de São Paulo, FAPESP

\section{ABSTRACT}

Chemical restraint in ostriches is usually required for short-time interventions. Thus, this study established and evaluated intravenous anesthetics formulated from commonly used drugs in order to accomplish total restraint on this species and allow painful procedures to be performed. Thirty male and female ostriches weighing from 40 to $90 \mathrm{~kg}$ were randomly distributed into five groups. Animals in Groups I, II and III were given acepromazine $(0.25 \mathrm{mg} / \mathrm{kg}$ i.m.) and those in Groups IV and $V$ were given xylazine $(1.0 \mathrm{mg} / \mathrm{kg}$ i.m.). The following drugs were administered intravenously 15 to 20 min later: Group I - propofol (4.0 $\mathrm{mg} / \mathrm{kg})$, Groups II and IV - ketamine $(5.0 \mathrm{mg} / \mathrm{kg})$ and diazepam $(0.25$ $\mathrm{mg} / \mathrm{kg}$ ), Groups III and V - tiletamine-zolazepam $(3.0 \mathrm{mg} / \mathrm{kg}$ ). All protocols have produced satisfactory results regarding total containment, muscular relaxation and maintenance of the evaluated parameters within a normal range.

\section{INTRODUCTION}

Ostrich farming has increased worldwide in the last years and also in Brazil. As a consequence of the higher numbers of raising facilities and the size of flocks, the demand for veterinary services has also increased. Therefore, improvements in contention techniques are required in order to allow the accomplishment of medical procedures.

According to Huchzermeyer (2000), the ostrich is the biggest bird in the world and it may be 2.75 meters high and weigh 150 kilograms. Ostriches belong to the ratite family of birds that comprises running birds. They cannot fly because of their rudimentary wings. Furthermore, ostriches have strong legs that may be used as defense in fights, by forward kicking.

Ostrich farming is a recent economical activity worldwide (Carrer \& Kornfeld, 1999). The first reference dates from the mid 1900's and have been reported in South Africa. According to Cornick-Seahorn (1996), this species preserves many characteristics from the wild animals and are highly susceptible to stress caused by handling and direct contact with humans, as usually occur during physical contention. Since ostriches are big and strong, physical contention may result in accidents leading to serious lesions not only in humans, but also in the birds.

The knowledge about ratite anesthesia refers mostly to non-captive, wildlife or even zoo animals, in contrast to little information available from ostriches reared in commercial production systems (CornickSeahorn, 1996).

In commercial rearing, chemical interventions and restraining are needed in many procedures such as sample collection and picking of feathers, and also in minor procedures, i.e., suturing and placement of esophageal probe. Extensive and invasive procedures like orthopedic 
Ciboto R, Cortopassi SRG, Lopes MAE, Carvalho RC, Baitelo CG
Comparison of chemical restraint techniques in ostrich (Struthio camelus) or intestinal surgeries are not frequently performed in this species. Therefore, the development of techniques that allow short-time chemical contention might be more useful than general anesthesia techniques.

According to Cornick-Seahorn (1996), the induction of anesthesia in ratites has been performed both IM and IV, and also by using inhalation anesthesia. The most used anesthetics are dissociative drugs, or yet associations between different drugs. The jugular, ulnar or metatarsal veins may be used in IV anesthesia.

The objective of this study was to perform a comparative analysis of different associations of anesthetic drugs in order to establish efficient techniques of short-term chemical contention to be used in commercially raised ostriches.

\section{MATERIAL AND METHODS}

Thirty male and female ostriches (Struthio camelus) were used. The birds were aged between eight and twelve months, and weighed between 40 and $90 \mathrm{~kg}$. The animals were bought from the ostrich farm Vereda dos Avestruzes, in Corumbataí, SP, Brazil.

The drugs used in the present study are listed below, together with the concentration:

- Propofol ${ }^{1}-10-\mathrm{ml}$ vial at $10 \mathrm{mg} / \mathrm{ml}$

- Acepromazine ${ }^{2}$ - $20-\mathrm{ml}$ vial at $10.0 \mathrm{mg} / \mathrm{ml}$

- Xylazine ${ }^{3}-20-\mathrm{ml}$ vial at $20 \mathrm{mg} / \mathrm{ml}$

- Diazepam ${ }^{4}-2-\mathrm{ml}$ vial at $5 \mathrm{mg} / \mathrm{ml}$

- Ketamine $-10-\mathrm{ml}$ vial at $100 \mathrm{mg} / \mathrm{ml}$

- Tiletamine-zolazepan ${ }^{6}$ - 5-ml vial at $50 \mathrm{mg} / \mathrm{ml}$

The birds were kept in pasture areas and were caught using rakes adequate for ostrich restraint and hooded. After transference to a smaller paddock and recovering from constraint stress, they were submitted to physical examination before anesthesia. Examination included mucosa evaluation, lung and heart auscultation, heart frequency, respiratory frequency and temperature measurement.

The birds were randomly distributed into five groups of six birds. Groups I, II and III were given acepromazine $(0.25 \mathrm{mg} / \mathrm{kg}$ IM) as pre-anesthesia drug, whereas Groups IV and $V$ were given xylazine $(1.0 \mathrm{mg} / \mathrm{kg} \mathrm{IM})$. After 15 minutes, a 16-G catheter was inserted into

1 Propofol - Cristália

2 Acepran $^{\circledR}$ a 1,0 \% - Univet S.A.

3 Rompun - Bayer

4 Diazepam - Cristália

5 Ketamine - Cristália

6 Telazol - Fort Dodge

7 GraphPad Instat - version 3.01 the ulnar vein of each bird and the drugs were administered as follows: Group I - propofol $4.0 \mathrm{mg} /$ $\mathrm{kg})$, Groups II and IV - ketamine $(5.0 \mathrm{mg} / \mathrm{kg}$ ) and diazepam $(0.25 \mathrm{mg} / \mathrm{kg})$, Groups III and V - tiletaminezolazepan $(3.0 \mathrm{mg} / \mathrm{kg})$.

The induction phase was determined as the time lag between drug administration and loss of postural control. The maintenance phase was considered as the time interval during which the bird was completely immobilized. The total recovery phase was defined as the period of time from the recovery of sensitivity and presence of voluntary movements until the birds were able to stand up. The birds were kept with hoods for 15 minutes afterwards.

Temperature, respiratory frequency and cardiac frequency were measured at five-minute intervals during the total anesthesia period until the beginning of the recovery phase. Blood pressure was measured during the period of complete immobilization.

Induction and recovery were classified as good if they were accomplished comfortably, rapidly and presented no risks to the animal and the assistants; reasonable, if there were no risks, but had lasting effects or a bad transition period; and bad when the animal and assistants were endangered, or if there was excessive distress and the drug had to be administered a second time.

Muscle relaxation was subjectively evaluated by the presence of resistance to flexing of limbs and the presence of non-induced muscle activity, according to the following score: 3 = intense (total muscular flaccidity); 2 = regular (discrete muscular tonus); 1 = mild (muscular tonus present); and $0=$ absent (contractions during handling).

The obtained values were evaluated by analysis of variance (ANOVA) using statistical software ${ }^{7}$, and the observation times within groups were compared using the Tukey's test. Statistical significance was set at $5 \%(p<0.05)$.

\section{RESULTS AND DISCUSSION}

Induction phase, induction quality, maintenance phase, and the time and quality of the recovery phase of the five groups are shown in Tables 1, 2 and 3.

It was observed that after acepromazine administration (Groups I, II and III), the parameters showed no changes and, in general, the animals were calmer and easily constrained, i.e., there was tranquilization. It was possible, for example, to immobilize the birds and perform the catheterization without muscle relaxation as reported by Kimminau (1993) and Cornick-Seahorn (1996). 
Ciboto R, Cortopassi SRG, Lopes MAE, Carvalho RC, Baitelo CG
Comparison of chemical restraint techniques in ostrich (Struthio camelus)
Table 1 - Mean values and standard deviations of the periods of induction, maintenance and recovery of Group I (acepromazine and propofol), Group II (acepromazin, ketamine and diazepam), Group III (acepromazine, tiletamine-zolazepam), Group IV (xylazine, ketamine and diazepam), and Group V (xylazine, tiletamine-zolazepan).

\begin{tabular}{lccc} 
& Induction (s) & Maintenance (min) & Recovery (min) \\
Group I & $16.60 \pm 10.32$ & $9.00 \pm 2.82$ & $17.66 \pm 6.05$ \\
Group II & $25.00 \pm 8.36$ & $8.80 \pm 2.64$ & $15.50 \pm 0.83$ \\
Group III & $16.67 \pm 10.80$ & $16.00 \pm 5.21$ & $26.83 \pm 6.76$ \\
Group IV & $28.33 \pm 8.16$ & $11.66 \pm 3.50$ & $15.33 \pm 0.81$ \\
Group V & $31.67 \pm 10.80$ & $18.66 \pm 2.33$ & $17.00 \pm 4.90$ \\
\hline
\end{tabular}

Xylazine was used as a pre-anesthesia drug in Groups IV and $V$ and produced good chemical immobilization, as seen by moderate muscle relaxation and sedation. Since xylazine is a sedative, the administration of greater doses than those used in the present study might intensify its effects. This might result in a more pronounced muscular relaxation and immobilization in ostriches as seen in other species, according to a previous report (Cullen et al., 1995).

In the present study, after xylazine was administered, the vital parameters were maintained without great variations, differently from what has been reported by Cullen et al. (1995) and CornickSeahorn (1996). The former authors have administered intravenous xylazine and observed bradycardia as well as sedation and mild ataxia. According to CornickSeahorn (1996), this drug might be used alone to induce sedation and immobilization in ostriches, or combined with ketamine to promote a more prolonged restraining; nevertheless, it should not be used in debilitated birds, due to its depressing cardio-respiratory effects.

Group I birds showed no significant changes ( $p>0.05$ ) in the heart or respiratory frequency during the anesthesia (propofol). Body temperature increased slightly at 15 and 20 min $(p<0.05)$. It was not possible to assess blood pressure in every evaluation time, because this procedure requires complete relaxation; the obtained results, however, were not significantly different ( $p>0.05)$. Muscle relaxation was intense in all animals at 15 minutes (100\%), in $66.7 \%$ of the birds at 20 min and four birds showed no relaxation at 25 min. It is known that propofol is a hypnotic agent with ultra-short action that is largely used in domestic animals because it provides good quality of induction and recovery. In the present study, the animals laid down in 10 to $20 \mathrm{~s}$ after intravenous administration of propofol. This induction was considered good and showed complete immobilization, with muscular relaxation and loss of standing posture. The maintenance period lasted $9 \mathrm{~min}$ in average; this is considered an ultra-short period, such as reported for other animal species. The cardio-respiratory parameters were stable and confirmed the results obtained by Mama et al. (1996) who described absence of cardio-respiratory alterations in barn owls ( Tyto alba) anesthetized with proporol.

Although no other studies on the administration of propofol in ostriches have been reported, the findings of the present study, together with results found in dogs and cats, indicate that propofol is a safe drug to induce anesthesia in this species. It might be useful in protocols of intravenous anesthesia, as far as it is used in short procedures, or yet, to develop techniques of continuous infusion. A negative aspect of this drug is that the volume to be administered was too large, because the concentration of the commercially available drug is $10 \mathrm{mg} / \mathrm{ml}$.

Ketamine and diazepam used after the acepromazin pre-anesthesia (Group II) was considered fast and easy to perform. During the maintenance period, the birds lay down and showed no voluntary movements or responses to external stimuli. Besides,

Table 2 - Quality of anesthesia induction as described in the text. Group I (acepromazin and propofol), Group II (acepromazin, ketamine and diazepam), Group III (acepromazin, tiletamine-zolazepam), Group IV (xylazine, ketamine and diazepam), and Group V (xylazine, tiletamine-zolazepan).

\begin{tabular}{|c|c|c|c|c|c|}
\hline Quality & Group I & Group II & Group III & Group IV & Group V \\
\hline Good & $100 \%$ & $100 \%$ & $100 \%$ & $83.33 \%$ & $100 \%$ \\
\hline Reasonable & $0.00 \%$ & $0.00 \%$ & $0.00 \%$ & $16.67 \%$ & $0.00 \%$ \\
\hline Bad & $0.00 \%$ & $0.00 \%$ & $0.00 \%$ & $0.00 \%$ & $0.00 \%$ \\
\hline
\end{tabular}

Table 3 - Quality of anesthesia recovery as described in the text. Group I (acepromazin and propofol), Group II (acepromazin, ketamine and diazepam), Group III (acepromazin, tiletamine-zolazepam), Group IV (xylazine, ketamine and diazepam), and Group V (xylazine, tiletamine-zolazepan.

\begin{tabular}{|c|c|c|c|c|c|}
\hline Quality & Group I & Group II & Group III & Group IV & Group V \\
\hline Good & $33.33 \%$ & $33.33 \%$ & $50.0 \%$ & $83.33 \%$ & $33.33 \%$ \\
\hline Reasonable & $50.00 \%$ & $33.33 \%$ & $16.77 \%$ & $16.67 \%$ & $66.67 \%$ \\
\hline Bad & $16.77 \%$ & $33.33 \%$ & $33.33 \%$ & $0.00 \%$ & $0.00 \%$ \\
\hline
\end{tabular}


Ciboto R, Cortopassi SRG, Lopes MAE, Carvalho RC, Baitelo CG

a good relaxation was obtained. The heart and respiratory frequencies, as well as mean blood pressure, showed no significant alterations during the evaluation periods ( $p>0.05)$. Body temperature increased slightly at 15 and $20 \mathrm{~min}(p<0.05)$. Muscle relaxation was intense in five animals at 15 minutes $(83.3 \%)$; at $20 \mathrm{~min}$, the animals showed intense $(66.7 \%)$ or significant muscle tonus (22.2\%); at $25 \mathrm{~min}$, two animals showed slight relaxation (33.3\%), two showed discrete relaxation $(33.3 \%)$ and the remaining showed no muscle relaxation (33.3\%). The maintenance period of this group was shorter in comparison to the other groups, lasting in average $8.8 \mathrm{~min}$. Therefore, this protocol is considered limited to be used in procedures with moderate duration. In contrast to the results obtained in the present study, the association between diazepam and ketamine was considered to produce good recovery, but unfeasible induction phase (CornickSeahorn \& Jensen, 1992).

Similarly to Group II, Group IV was given diazepam and ketamine. The cardiac frequency and the mean blood pressure showed no significant alterations $(p<0.05)$. As for the respiratory frequency, an increase was observed at 15 and $20 \mathrm{~min}$. This was different from the basal frequency $(p<0.05)$ similarly to the results reported by Barreiro et al. (2002). Body temperature was slightly increased at 10,15 and $20 \mathrm{~min}$, and significantly different from the basal temperature $(p<0.05)$. The degree of muscle relaxation was intense in $66.6 \%$ of the birds at 15 and 20 minutes, whereas at 25 minutes only $16.7 \%$ showed intense relaxation, $50 \%$ showed discrete relaxation and the remaining $33.3 \%$ had significant muscle tonus.

The average maintenance period in Group III was $16 \mathrm{~min}$ and there was muscle relaxation. This finding is not in accordance to previous results in which immobilization was not achieved after intramuscular injection of tiletamine-zolazepan (Van Heerden \& Keffen, 1991). Relaxation was intense in all animals at 15 and $20 \mathrm{~min}(100 \%)$; at $25 \mathrm{~min}, 66.6 \%$ showed intense relaxation whereas the others $(33.3 \%)$ had significant muscular tonus; at 30 min, only two birds showed intense relaxation (33.3\%) and the remaining showed discrete relaxation (66.7\%). There were no differences ( $p>0.05)$ in the cardiac and respiratory frequencies, body temperature or blood pressure.

In Group V, the association between tiletaminezolazepan was administered after pre-anesthesia with xylazine and resulted in good induction, loss of standing posture and muscular relaxation. The cardiac frequency reduced at $20 \mathrm{~min}(\mathrm{p}<0.05)$. However, such
Comparison of chemical restraint techniques in ostrich (Struthio camelus)

variation should be disregarded, since the birds of this group had basal values considered high for this species. This is probably due to the manipulation that was performed during constraint and other external factors, which resulted in disturbance during the trial. There were no significant changes $(p<0.05)$ in the respiratory frequency, body temperature and blood pressure during assessments. Muscle relaxation was intense in $66.7 \%$, regular in $16.7 \%$ and mild in $16.7 \%$ of the birds at $15 \mathrm{~min}$; at $20 \mathrm{~min}$, only $16.7 \%$ of the birds had mild relaxation and the others showed intense muscle relaxation; at $25 \mathrm{~min}, 66.7 \%$ showed discrete relaxation and the remaining birds, intense relaxation.

The induction periods of the different protocols showed no significant differences. The analysis of the maintenance period values indicates that the protocols were different. Group $\vee$ showed longer maintenance period in comparison to Groups I, II and IV $(p<0.05)$. Similarly, the maintenance period of Group III was greater than that obtained in Groups I and II. Longer maintenance period was produced by the protocol xylazine-tiletamine-zolazepan, which was provided by the quality of muscle relaxation of the $\alpha-2$ adrenergic agonist and the greater effectiveness of the dissociative agent.

The recuperation of animals in Groups II and III had to be monitored by the assistants due to the variable behavior between animals. This result corroborates the report of Van Heerden \& Keffen (1991), in which sudden movements of the head, neck and limbs were observed with lesions during the recovery from anesthesia performed with tiletamine-zolazepan. On the other hand, quiet recovery has been reported after anesthesia with an association of three drugs, phenotiazine, benzodiazepine and a dissociative agent (Barreiro et al., 2002). Group III showed longer recovery periods than other groups $(p<0.05)$, probably due to greater half-life of the used agents (acepromazine/ tiletamine-zolazepan).

The protocols were similar in regard to the maintenance of parameters such as cardiac and respiratory frequencies, temperature and blood pressure. All of them resulted in irrelevant alterations in comparison to the basal values. Moreover, during the anesthesia period, the animals showed similar behavior of decubitus and relaxation, besides the absence of response to stimuli.

Unfortunately it was not possible to obtain more conclusive data in regard to blood pressure, since the measurement of this parameter requires complete muscle relaxation. Therefore, basal values were very 
difficult to be obtained and it is believed that protocols that promote longer action periods, as well as relaxation, allow easier and more accurate measurements.

\section{CONCLUSION}

The present study introduces five anesthetic protocols for ostriches that might be used by veterinarians. The protocols comprise easily accessible drugs that promote good quality anesthesia, with safe induction and recovery, and different times of induction, maintenance and recovery.

Both xylazine and acepromazine might be used as pre-anesthetic drugs with efficiency and similar results.

Surely, in regard to the protocols, the main differences were generally due to significant variation in the periods of maintenance and recovery.

In conclusion, the description of the response profiles produced by the protocols evaluated in the present study enables veterinarians to choose a protocol according to a given situation, depending on the relaxation degree, immobilization time and drug availability.

\section{REFERENCES}

Barreiro RH, Massone F, Balieiro JCC, Elmôr RA, Hatschbach E, Lobo MAMS. Associação de levomepromazina, midazolam e ketamine ou levomepromazina, diazepam e ketamine pela via intravenosa em avestruz (Struthio camelus). Revista Brasileira de Ciência Veterinária 2002; 9(1):148-50.

Carrer CC, Kornfeld ME. A criação de avestruzes no Brasil. Pirassununga: Brasil Ostrich Comercial 1999; 25-48.

Cornick-Seahorn JL, Anesthesiology of Ratites. In: Tully TN, Shane SM, editors. Ratite management and surgery. Malabar: Krieger Publishing; 1996.

Cornick-Seahorn JL, Jensen J. Anesthetic management of ostriches. Journal of the American Veterinary Medical Association 1992; 200: 1661-6.

Cullen LK, Gorke MA, Swan RA, Clark WT, Nandapi D \& Colbourne $C$. Ostrich anesthesia: xylazine premedication followed by alphaxalone/alphadolone and isofluorane. Australian Veterinary Journal 1995; 72: 153-4.

Huchzermeyer FW. Doenças de avestruzes e outras ratitas, Jaboticabal: Funep; 2000.

Kimminau KM. Introduction the ostrich. Veterinary Technician 1993; 14:459-67.

Mama KR, Phillips LG, Pascoe PJ. Use of propofol for induction and maintenance of anesthesia in a barn owl (Tyto alba) undergoing tracheal resection. Journal Zoological Wildlife Medicine 1996; 27(3): 397-401.

Van Heerden J, Keffen RH. A preliminary investigation into the immobilising potential of a tiletamine/zolazepam mixture, metomidate, a metomidate and azaperone combination and metomidine in ostriches ( $S$. camelus). Journal South African Veterinary Association 1991; 62:114-7. 